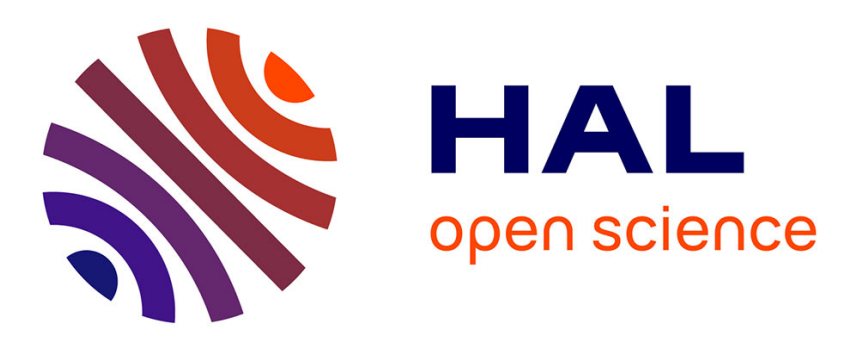

\title{
Damping of a pendulum: An experimental test of the Stokesian unsteady friction force on a cylinder
}

Gilles Dolfo, Jacques Vigué, Daniel Lhuillier

\section{To cite this version:}

Gilles Dolfo, Jacques Vigué, Daniel Lhuillier. Damping of a pendulum: An experimental test of the Stokesian unsteady friction force on a cylinder. Physical Review Fluids, 2021, 6 (10), pp.104101. 10.1103/PhysRevFluids.6.104101 . hal-03442282

HAL Id: hal-03442282

https://hal.science/hal-03442282

Submitted on 25 Nov 2021

HAL is a multi-disciplinary open access archive for the deposit and dissemination of scientific research documents, whether they are published or not. The documents may come from teaching and research institutions in France or abroad, or from public or private research centers.
L'archive ouverte pluridisciplinaire HAL, est destinée au dépôt et à la diffusion de documents scientifiques de niveau recherche, publiés ou non, émanant des établissements d'enseignement et de recherche français ou étrangers, des laboratoires publics ou privés. 


\title{
Damping of a pendulum: An experimental test of the Stokesian unsteady friction force on a cylinder
}

\author{
G. Dolfo* and J. Vigué $\odot$ \\ Laboratoire Collisions Agrégats Réactivité-FERMI \\ Université de Toulouse-UPS and CNRS UMR 5589, 31062 Toulouse, France \\ D. Lhuillier \\ Sorbonne Université, CNRS, Institut Jean Le Rond d'Alembert, \\ 4 place Jussieu, Boite 162, 75252 PARIS CEDEX 05, France
}

(Received 6 November 2020; accepted 5 October 2021; published 28 October 2021)

\begin{abstract}
In 1851, Stokes calculated the force exerted by a viscous fluid on an infinite cylinder in oscillating motion. Although the calculation was restricted to vanishingly small Reynolds numbers Re, most of the experimental tests performed up to now have been made with $\operatorname{Re}>1$. Here we present a series of experiments involving a cylindrical pendulum oscillating in air at different pressures and with $\operatorname{Re}<1$. We deduce the unsteady friction force from the measurement of the damping time of the oscillations. We compare our experimental results for the unsteady force (a) to Stokes' predictions for an infinite cylinder and (b) to predictions for finite-length cylinders, using an expression of the unsteady force derived from the works of Lawrence and Weinbaum and Loewenberg. The agreement is quite satisfactory and proves that macroscopic objects like a pendulum can be used as probes of the Stokesian flow regime.
\end{abstract}

DOI: 10.1103/PhysRevFluids.6.104101

\section{INTRODUCTION}

In 1851 Stokes [1] calculated the force exerted by a viscous fluid on a sphere and on an infinite cylinder, in steady as well as in oscillating motion. Concerning the sphere, his calculations were successful for both kinds of motion. For the steady motion of the cylinder, however, Stokes faced a difficulty which is now coined as the "Stokes paradox." Despite that failure for steady flows, Stokes succeeded in obtaining the unsteady force acting on an oscillating infinite cylinder. More recently, a new branch of fluid dynamics has arisen - the study of suspensions of particles at low Reynolds numbers. One of the challenges has been to model the force on oscillating particles. Lawrence and Weinbaum $(\mathrm{L} \& \mathrm{~W})$ [2] proposed an expression of the unsteady force acting on spheroids while Loewenberg $[3,4]$ adapted the $\mathrm{L} \& \mathrm{~W}$ expression to finite-length cylinders. A last question remains, however: Can the unsteady force on an infinite cylinder be obtained from the unsteady force acting on finite cylinders with higher and higher aspect ratios?

Several types of experiments have been used to measure the unsteady force on an oscillating cylinder. Here are the papers we are aware of, without any claim for exhaustivity:

(1) Experiments with a torsion pendulum carrying two cylindrical rods immersed in a liquid. The first experiments of this type were done in 1800 by Coulomb [5] and similar experiments were done in 1886 by Tomlinson [6] and in 1972 by Williams and Hussey [7,8].

\footnotetext{
*gilles.dolfo@wanadoo.fr
} 
TABLE I. This table synthesizes information concerning the experiments of Martin [12], of Stuart and Woodgate [10], and of Williams and Hussey [7,8]. For each experiment, we give the fluid used, the cylinder radius $R$ in millimeters, the aspect ratio $\phi=L /(2 R)$ (where $L$ is the cylinder length), the oscillation frequency $\omega /(2 \pi)$ in hertz, the values of the Stokes number defined by $\mathrm{St} \equiv 4 R^{2} / \delta^{2}$, of the Reynolds number defined by $\operatorname{Re} \equiv 2 R \rho U_{0} / \eta=4 x_{0} R / \delta^{2}$, and of the ratios $x_{0} / R$ and $x_{0} / \delta$ [where $\delta$ is the viscous penetration depth defined after Eq. (1)]. In the case of Martin's experiments, we give only the values corresponding to the largest studied frequency, the lowest frequency being $250 \mathrm{~Hz}$

\begin{tabular}{|c|c|c|c|c|c|}
\hline Author & & Martin & & Stuart & Williams and Hussey \\
\hline Fluid & & Water & & Air & Various liquids \\
\hline$R$ & 0.15 & 0.255 & 0.405 & 25.4 & $1.59-6.35$ \\
\hline$\phi$ & $\sim 830$ & $\sim 500$ & $\sim 310$ & 24 & $\sim 8$ to $\sim 31$ \\
\hline$\omega /(2 \pi)$ & & 1600 & & 0.690 & $0.06-0.2$ \\
\hline St & 424 & 1259 & 3113 & 384 & $0.24-49.0$ \\
\hline $\operatorname{Re}$ & 152 & 262 & 414 & 68 & $0.17-39.0$ \\
\hline$x_{0} / R$ & 0.36 & 0.21 & 0.13 & 0.18 & $0.4-2.5$ \\
\hline$x_{0} / \delta$ & & 3.7 & & 1.7 & $0.15-2.79$ \\
\hline
\end{tabular}

(2) Experiments with a gravity pendulum oscillating in air by Baily [9] in 1832 and by Stuart and Woodgate [10] in 1955; the experiment described in the present paper belongs to this type.

(3) Experiments with vibrating strings by Laird [11] in 1898 and by Martin [12] in 1925.

(4) Berg et al. [13] have studied the friction force on an oscillating cylinder and its variations with the oscillation amplitude, using experiments and numerical calculations.

We give more details on these experiments in the Supplemental Material [14]. Almost all of the quoted experiments were operated with rather large values of the Reynolds number Re (cf. Table I) and moderate to large values of the cylinder aspect ratio. Despite this, most of the authors claimed a rather good agreement with Stokes predictions which were obtained for a vanishing Reynolds number and an infinite cylinder. If one can admit that a very long cylinder behaves approximately like an infinite one, the true role of the Reynolds number is more puzzling and this is the reason why we strived to build an experiment operating with the smallest possible Re.

Our paper is organized as follows. At first we recall the results obtained by Stokes for an infinite cylinder and, extending results by Lawrence and Weinbaum [2], we propose an expression of the unsteady force acting on cylinders of high aspect ratios. Then we present our experiment and the way we relate the damping time of a pendulum to the unsteady force exerted on it. We then present the experimental results and compare them to the predictions for infinite and finite-length cylinders. Finally, with consideration of the magnitude of the Stokes number, we will (tentatively) explain why experimental results obtained with finite Reynolds numbers can agree with theoretical predictions for vanishing Re.

\section{THE UNSTEADY FORCE ON CYLINDERS AT Re $=0$}

In this section, we recall the result obtained by Stokes [1] for an infinite cylinder and the result obtained by Lawrence and Weinbaum [2] and Loewenberg [3,4] for spheroids and finite cylinders. Finally we propose for the unsteady force an expression that interpolates between finite and infinite cylinders.

\section{A. Transverse periodic motion of an infinite cylinder}

An infinite cylinder of radius $R$ oscillates in a fluid with a displacement $x(t)=x_{0} \sin (\omega t)$ perpendicular to the cylinder axis. The force per unit length exerted by the fluid on the cylinder 
is given by [1]

$$
\frac{d F^{\text {Stokes }}}{d l}=-2 \pi \eta\left[K^{\prime} U_{0} \cos (\omega t)-K U_{0} \sin (\omega t)\right],
$$

where $\eta$ is the fluid viscosity and $U_{0}=\omega x_{0}$. The two components of the force are of different natures. The term in $K$, proportional to the acceleration, describes an increase of the cylinder inertia due to the interaction with the fluid and it is called the added mass effect. In the case of a pendulum, its consequence is a modification of its period but this term does not induce any damping effect. The term in $K^{\prime}$ is proportional to the velocity and it describes the friction force which produces damping of the pendulum oscillation. For an infinite cylinder both coefficients $K$ and $K^{\prime}$ are functions of one single variable which is the ratio $R / \delta$ where $\delta=\sqrt{2 \eta / \rho \omega}$ is the viscous penetration depth and $\rho$ is the mass per unit volume of the fluid. Stokes obtained $K$ and $K^{\prime}$ in the form of a development in series for the two limiting cases of low frequencies $(R / \delta \ll 1)$ and high frequencies $(R / \delta \gg 1)$. The first terms of these series are

$$
\begin{aligned}
K= & \frac{\pi / 2}{[\ln (R / \delta)]^{2}}+\cdots, \quad K^{\prime}=-\frac{2}{\ln (R / \delta)}+\cdots \\
& \text { if } R / \delta \ll 1, \\
K= & \left(\frac{R}{\delta}\right)^{2}+2 \frac{R}{\delta}+\cdots, \quad K^{\prime}=2 \frac{R}{\delta}+1+\cdots, \\
& \text { if } R / \delta \gg 1 .
\end{aligned}
$$

The complete (and compact) expressions for $K$ and $K^{\prime}$ were obtained more than a century later $[15,16]$, in a complex-valued form

$$
K-i K^{\prime}=\left(\frac{R}{\delta}\right)^{2}+2 \lambda \frac{K_{1}(i \lambda)}{K_{0}(i \lambda)},
$$

where $\lambda=(1-i) R / \delta$ and $K_{n}$ is the modified Bessel function of order $n$.

The (unexpected) logarithmic collapse (2) of the coefficients $K$ and $K^{\prime}$ for very low frequencies is presumably related to the so-called Stokes paradox observed for the steady motion of the infinite cylinder. While these low-frequency results are questionable, the high-frequency limit (3) stands on much firmer ground and is the starting point for expressions of $K$ and $K^{\prime}$ which hold in a much broader range of $R / \delta$. For example, one can write for $R / \delta>0.2$

$$
\begin{aligned}
K & \approx\left(\frac{R}{\delta}\right)^{2}+2 \frac{R}{\delta}+\frac{R / \delta}{8[(R / \delta)+0.340]^{2}}, \\
K^{\prime} & \approx 2 \frac{R}{\delta}+1-\frac{R / \delta}{8[(R / \delta)+0.107]^{2}},
\end{aligned}
$$

with deviations less than $1 \%$ from the general result (4).

\section{B. Transverse periodic motion of a finite cylinder}

For prolate spheroids in transverse periodic motion Lawrence and Weinbaum [2] proposed a general expression of the force, supposed to hold for all frequencies:

$$
\begin{gathered}
\frac{F}{\eta R U_{0}}=-\left[R_{\perp}^{0}+B_{\perp}^{\infty} \frac{R}{\delta}+\left(B_{\perp}^{0}-B_{\perp}^{\infty}\right) H\left(\frac{R}{\delta}\right)\right] \cos (\omega t) \\
+\left[2 M_{\perp}^{A} \frac{R^{2}}{\delta^{2}}+B_{\perp}^{\infty} \frac{R}{\delta}+\left(B_{\perp}^{0}-B_{\perp}^{\infty}\right) G\left(\frac{R}{\delta}\right)\right] \sin (\omega t) .
\end{gathered}
$$


The coefficients $R_{\perp}^{0}, B_{\perp}^{\infty}$, and $M_{\perp}^{A}$ depict the steady drag, Basset and added-mass forces while $B_{\perp}^{0}=$ $\left(R_{\perp}^{0}\right)^{2} / 6 \pi$ depicts the unsteady drag force at low frequency. These four coefficients are functions of the aspect ratio of the spheroid, and the two interpolating functions $H$ and $G$ are defined as

$$
H(x)=\frac{2 x^{2}+x}{2 x^{2}+2 x+1}, \quad G(x)=\frac{x}{2 x^{2}+2 x+1} .
$$

The same general expression of the unsteady force was also adopted by Loewenberg $[3,4]$ for cylinders of finite length. It appeared that the L\&W force gives satisfactory predictions for prolate spheroids and cylinders of moderate aspect ratio $(1<\phi<10)$ but not for larger ones. The reason is clearly the very large values of the unsteady drag coefficient $B_{\perp}^{0}(\phi)$ for large aspect ratios which, when using the L\&W expression, leads to an overestimate of the force acting on elongated particles at high frequencies. We would like to modify the L\&W expression so as to obtain a force which would hold for all aspect ratios and we focus on the case of cylinders of aspect ratio $\phi=L /(2 R)$. When this aspect ratio is very large, we notice that $d F^{\text {Stokes }} / d l=F / L=F /(2 R \phi)$ so that one is led to rewrite the Stokesian force (1) in a form similar to the L\&W force (6)

$$
\frac{F}{\eta R U_{0}}=-4 \pi \phi\left[K^{\prime} \cos (\omega t)-K \sin (\omega t)\right]
$$

with $K$ and $K^{\prime}$ now depending not only on $(R / \delta)$ but also on the aspect ratio $\phi$. And to agree with Stokes' main result, we require that $K$ and $K^{\prime}$ must tend to the nondiverging limit values (5) when the aspect ratio increases to infinity. Is there a possible interpolation between the Stokesian unsteady force for infinite aspect ratio and high frequencies and the L\&W force which is known to hold for small aspect ratios and all frequencies? We could obtain a satisfactory interpolation when writing

$$
\begin{gathered}
4 \pi \phi K=2 M_{\perp}^{A} \frac{R^{2}}{\delta^{2}}+B_{\perp}^{\infty} \frac{R}{\delta}+4 \pi \phi G\left[\frac{B_{\perp}^{0}-B_{\perp}^{\infty}}{4 \pi \phi} \frac{R}{\delta}\right], \\
4 \pi \phi K^{\prime}=R_{\perp}^{0}+B_{\perp}^{\infty} \frac{R}{\delta}+4 \pi \phi H\left[\frac{B_{\perp}^{0}-B_{\perp}^{\infty}}{4 \pi \phi} \frac{R}{\delta}\right],
\end{gathered}
$$

where $G(x)$ and $H(x)$ are the $\mathrm{L} \& \mathrm{~W}$ interpolating functions defined in (7). The main difference is that $\left(B_{\perp}^{0}-B_{\perp}^{\infty}\right)(R / \delta)$ replaces $(R / \delta)$ as the argument of these interpolating functions. The main consequence is that the force acting on elongated cylinders at high frequency has now the correct order of magnitude and the Stokesian force will be obtained for an infinite aspect ratio. Since the factor $4 \pi \phi$ is present everywhere, in what follows we rewrite the above expressions for $K$ and $K^{\prime}$ as

$$
\begin{gathered}
K=2 m_{\perp}^{A} \frac{R^{2}}{\delta^{2}}+b_{\perp}^{\infty} \frac{R}{\delta}+G\left[\left(b_{\perp}^{0}-b_{\perp}^{\infty}\right) \frac{R}{\delta}\right], \\
K^{\prime}=r_{\perp}^{0}+b_{\perp}^{\infty} \frac{R}{\delta}+H\left[\left(b_{\perp}^{0}-b_{\perp}^{\infty}\right) \frac{R}{\delta}\right]
\end{gathered}
$$

where $\left(r_{\perp}^{0}, b_{\perp}^{0}, b_{\perp}^{\infty}, m_{\perp}^{A}\right)=\left(R_{\perp}^{0}, B_{\perp}^{0}, B_{\perp}^{\infty}, M_{\perp}^{A}\right) /(4 \pi \phi)$. Useful information concerning the dependence of these coefficients on aspect ratio was obtained by Loewenberg [3,4]. For aspect ratios $\phi>10$ (as in our experiments) the coefficients $b_{\perp}^{\infty}$ and $m_{\perp}^{A}$ do not vary much as compared to their value for an infinite cylinder $\left[b_{\perp}^{\infty}(\infty)=2\right.$ and $\left.m_{\perp}^{A}(\infty)=0.5\right]$ since for $\phi>10$

$$
2<b_{\perp}^{\infty}(\phi)<2.013, \quad 0.468<m_{\perp}^{A}(\phi)<0.5
$$

while the steady and unsteady drag coefficients for $\phi>10$ are $[3,4]$

$$
r_{\perp}^{0}(\phi)=\frac{2+0.614 / \ln (2 \phi)}{\frac{1}{2}+\ln (2 \phi)}+\frac{0.238}{[\ln (2 \phi)]^{3}},
$$




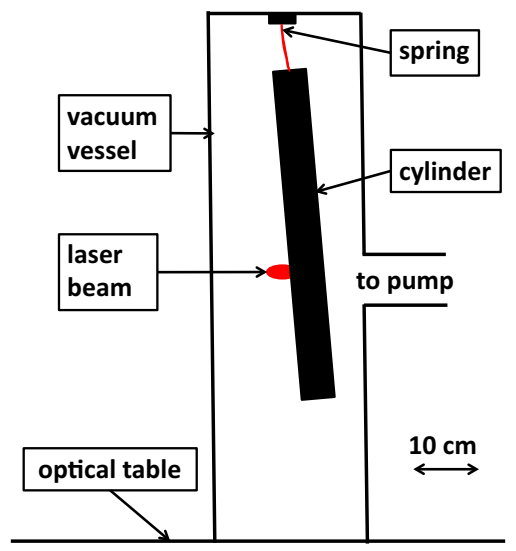

FIG. 1. Schematic drawing of our setup, with the $R \approx 19 \mathrm{~mm}$ cylinder. The spring thickness, the oscillation amplitude, and the size of the laser beam of the shadow detector have been grossly exaggerated in order to be visible. The double arrow gives the length scale.

$$
b_{\perp}^{0}(\phi)=\frac{2 \phi}{3}\left(r_{\perp}^{0}\right)^{2}
$$

Note that the steady drag coefficient $r_{\perp}^{0}$ slowly decreases to zero (like $1 / \ln \phi$ ) when the aspect ratio increases to infinity while the unsteady drag coefficient $b_{\perp}^{0}$ increases to infinity like $\phi /(\ln \phi)^{2}$.

The results of our experiments will concern the high-frequency limit [now defined as $(R / \delta) \gg$ $\left.1 /\left(b_{\perp}^{0}-b_{\perp}^{\infty}\right)\right]$ and, for future use, we write below the high-frequency asymptotic forms for $K$ and $K^{\prime}$ :

$$
\begin{gathered}
K=2 m_{\perp}^{A}(\phi) \frac{R^{2}}{\delta^{2}}+b_{\perp}^{\infty}(\phi) \frac{R}{\delta}, \\
K^{\prime}=r_{\perp}^{0}(\phi)+b_{\perp}^{\infty}(\phi) \frac{R}{\delta}+1 .
\end{gathered}
$$

Consequently for an infinite cylinder and high frequencies, $K=(R / \delta)^{2}+2 R / \delta$ and $K^{\prime}=2 R / \delta+1$ in conformity with (3). Note, however, that a difference exists with the more complete Stokesian result (5). The last term of (5) is certainly a small correction in the high-frequency domain. However, we could not find a plausible $\phi$ dependence for it and we will limit to (16) and (17) the comparison between theory and experiment.

\section{OUR EXPERIMENTS}

\section{A. Experimental setup}

Our setup is very similar to the one devised by Stuart and Woodgate [10]: a pendulum made of a long cylinder oscillates in a pressurized vessel and we measure the damping of its oscillations for various air pressures. The decrease of the oscillation amplitude is measured by a shadow detector with a submicrometer sensitivity. Figure 1 presents a schematic drawing of our setup. We have used two pendulums, both being made of an aluminum alloy (2017A), with a length $\sim 500 \mathrm{~mm}$. The larger cylinder is hollow, with an external radius $R \approx 19 \mathrm{~mm}$ and an internal radius $R^{\text {int }} \approx 16 \mathrm{~mm}$. The smaller mass of a hollow rod reduces the damping time and the duration of an experiment. The thinner cylinder is a rod of radius $R \approx 5 \mathrm{~mm}$. These pendulums are suspended by a spring made of a rectangular thin sheet of brass clamped, at its upper end, on the top flange of the pressurized tank 
and, at its lower end, on the pendulum. The dimensions, masses, and other parameters of the two pendulums are collected in the Supplemental Material [14].

The pressurized vessel is a cylinder with a $125 \mathrm{~mm}$ internal radius and an $800 \mathrm{~mm}$ height. It is fixed on a heavy optical table and connected by a flexible pipe to an Edwards T85 pumping station, made of a dry primary pump and a turbo pump. The residual pressure $P$ achieved after $24 \mathrm{~h}$ of pumping is $P<10^{-5} \mathrm{mbar}$, measured by a Bayard-Alpert gauge. For the experiments, the pressure, always larger than 9 mbar, is measured by a diaphragm gauge (Leybold Ceravac), with a $\pm 0.2 \mathrm{mbar}$ uncertainty mainly due to a slow drift of the gauge zero.

The shadow detector has been previously described $[17,18]$ : its deviation from linearity is smaller than a few percent for a pendulum displacement $x_{0}<0.5 \mathrm{~mm}$. The scale factor is $\sim 10^{3} \mathrm{~V} / \mathrm{m}$ and the noise level $\sim 3 \times 10^{-4} \mathrm{~V} / \sqrt{\mathrm{Hz}}$ at the pendulum frequency, corresponding to a sensitivity limit $\sim 3 \times 10^{-7} \mathrm{~m} / \sqrt{\mathrm{Hz}}$.

\section{B. Data recording}

In a first step we measure the pendulum resonant frequency, near $0.8 \mathrm{~Hz}$, with a $1 \mathrm{mHz}$ accuracy using the Fourier transform function of a digital oscilloscope with a 200-s-long record. Then, at the beginning of each measurement, the pendulum oscillation is excited by the force due to a magnetic field gradient acting on a NdFeB magnet of dimensions $2 \times 3 \times 10 \mathrm{~mm}^{3}$ fixed at the bottom of the pendulum. The field is produced by a coil located outside the nonmagnetic stainless-steel vessel and the voltage applied to the coil oscillates at the measured resonant frequency. The amplitude of the excitation voltage and the duration of the excitation are chosen to produce an oscillation amplitude $\approx 0.5 \mathrm{~mm}$. When this amplitude is reached, the voltage applied to the coil is suppressed and the pendulum oscillates freely. For the experiments with the $R \approx 19 \mathrm{~mm}$ pendulum at a pressure $P>600$ mbar, the achieved oscillation amplitude was only $0.3-0.4 \mathrm{~mm}$ limited by the larger friction force but, for all experiments, we use $x_{0}=0.5 \mathrm{~mm}$ to calculate the Reynolds number Re, and the ratios $x_{0} / R$ and $x_{0} / \delta$.

We now describe our protocol for a series of experiments. We first pump down the pressurized vessel to a pressure $P<10^{-5} \mathrm{mbar}$. Then, we let some air enter and we make a series of measurements of the damping time at some controlled pressure. We then let some more air enter and we start a new series of measurements at a different pressure, and so on. As the oscillation amplitude is very small, it is perturbed if somebody touches the optical table. In order to avoid such perturbations, all the experiments are done with nobody in the room. Immediately after the end of the excitation period, the data recording starts. The shadow detector measures the pendulum position $x(t)$. Its signal and the Ceravac gauge signal are digitized at $40 \mathrm{~Hz}$ by a PXI 16-bit digitizer. The process then starts again until we get five to ten records for the same pressure.

\section{RELATION BETWEEN THE FRICTION FORCE AND THE DAMPING TIME CONSTANT}

\section{The pendulum motion}

A pendulum suspended by a spring [19] has two modes of oscillation, which can be described as harmonic oscillators in the limit of small amplitudes: a long-period mode, corresponding approximately to the usual pendular motion and a short-period mode, corresponding approximately to a rotation of the pendulum body around its center of mass. The present experiments are all made with the long-period mode which can be described by a mass-spring system with a displacement $x(t)$ obeying the differential equation

$$
M \frac{d^{2} x}{d t^{2}}+k_{s} x=f^{h}(t)+f^{n h}(t)
$$

$M$ is the mass of the cylinder. For a hollow cylinder of internal radius $R_{\text {int }}$, of external radius $R$, and length $L, M=\rho_{p} \pi\left(R^{2}-R_{\text {int }}^{2}\right) L$, where $\rho_{p}$ is the mass per unit volume, and $k_{s}$ is the effective spring constant, which is the sum of the contributions due to gravity and to the suspending spring itself. $f^{h}$ 
is the hydrodynamic force exerted by the ambient air on the cylinder of length $L$ :

$$
f^{h}=-2 \pi \eta L\left[K^{\prime} \frac{d x}{d t}+\frac{K}{\omega} \frac{d^{2} x}{d t^{2}}\right],
$$

while $f^{n h}$ collects all damping forces of nonhydrodynamic origin. Among the physical phenomena that can contribute to $f^{n h}$ are (i) the anelastic effect in the suspension spring (this is a thermal effect explained theoretically by Zener [20,21]); (ii) the recoil losses due to a deformation of the pendulum support not in phase with the force exerted by the pendulum [22]; and (iii) the eddy currents induced by the magnet carried by the pendulum in the conducting materials of the pressurized tank. We assume that all these different phenomena can be described by a damping force proportional to the velocity

$$
f^{n h}=-\zeta^{n h} \frac{d x}{d t}
$$

The resulting equation of motion is

$$
\begin{aligned}
& \left(M+M^{h}\right) \frac{d^{2} x}{d t^{2}}+\left(\zeta^{h}+\zeta^{n h}\right) \frac{d x}{d t}+k_{s} x=0, \\
& M^{h}=\rho\left(\pi R^{2} L\right)\left(K \frac{\delta^{2}}{R^{2}}\right), \quad \zeta^{h}=2 \pi \eta L K^{\prime} .
\end{aligned}
$$

Note that the relative importance of the added mass can be estimated by the ratio

$$
\frac{M^{h}}{M}=\frac{\rho R^{2}}{\rho_{p}\left(R^{2}-R_{\mathrm{int}}^{2}\right)}\left[2 m_{\perp}^{A}(\phi)+b_{\perp}^{\infty}(\phi) \frac{\delta}{R}\right],
$$

where expression (16) for $K$ was taken into account. For each of the two cylinders the right-hand side is a function of the gas pressure (which acts on both $\rho$ and $\delta$ ) and for the domain of pressure that we investigated, we checked that $M^{h} / M<1.2 \times 10^{-3}$ for the $R=19 \mathrm{~mm}$ pendulum and $M^{h} / M<$ $0.5 \times 10^{-3}$ for the $R=5 \mathrm{~mm}$ one. The contribution of the added mass is thus negligible and $M^{h}$ will be neglected in what follows.

Our experiments give direct access to the amplitude $a(t)$ of the oscillating motion $x(t)=$ $a(t) \sin (\omega t)$. According to the above mass-spring equation, the amplitude decreases exponentially

$$
a(t)=a(0) \exp (-t / \tau)
$$

with the damping time $\tau$ defined as

$$
\frac{1}{\tau}=\frac{\left(\zeta^{h}+\zeta^{n h}\right)}{2 M}=\frac{1}{\tau^{h}}+\frac{1}{\tau^{n h}}
$$

Experiments at very low pressures $P<10^{-5}$ mbar have been made to measure the nonhydrodynamic damping time. This is possible because these nonhydrodynamic effects are independent of the gas density. For such low pressures, the mean free path is larger than the size of the pressurized tank and, according to formulas found in Cagnoli et al. [22], the collision-induced damping time is inversely proportional to the pressure. This damping time is of the order of $\tau^{h} \approx 3 \times 10^{7} \mathrm{~s}$ for $P=10^{-5}$ mbar. This rarefied gas damping time is considerably larger than the experimental value $\tau^{\text {expt }} \approx 2 \times 10^{5}$ s measured for $P<10^{-5}$ mbar. More details can be found in the Supplemental Material [14]. As a consequence, we will assume that the nonhydrodynamic damping time is $\tau^{n h}=2 \times 10^{5} \mathrm{~s}$ and we can calculate $\tau^{h}$ by the following equation:

$$
\frac{1}{\tau^{h}}=\frac{1}{\tau^{\operatorname{expt}}}-\frac{1}{\tau^{n h}}
$$




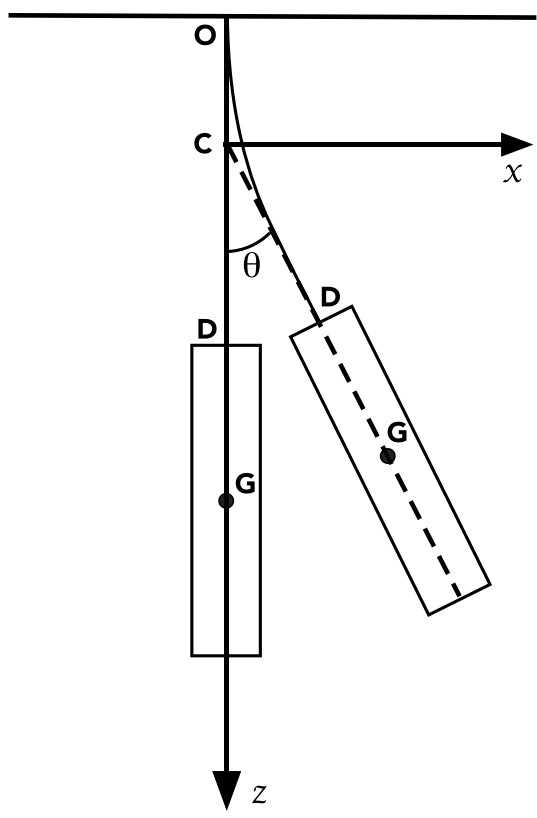

FIG. 2. Schematic drawing of the pendulum (not to scale). The spring and the cylindrical body are represented in two positions. The rest position, $\theta=0$, is used to calculate the moment of inertia for rotation around $C$ and the position with $\theta \neq 0$ exhibits the shape of the spring and the center of rotation $C$ of the pendulum body. $O$ is the upper end of the spring where it is clamped in the support and $D$ its lower end, where it is clamped in the pendulum body, while $G$ is the center of mass of the pendulum body. The length $L_{1}$ is equal to $C D$. The $x$ axis, the $z$ axis, and the oscillation angle $\theta$ are represented. The angle $\theta$ has been grossly exaggerated as, in our experiments, it never exceeds $1 \mathrm{mrad}$.

The product $\eta K^{\prime}$ is then given as

$$
\eta K^{\prime}=\frac{M}{\pi L} \frac{1}{\tau^{h}}
$$

where $M=\rho_{p} \pi\left(R^{2}-R_{\text {int }}^{2}\right) L$.

It is clear that the true pendular motion is an angular oscillation around some center of rotation $C$ (see Fig. 2 ) and not a uniform oscillatory motion as described in (21). In the Supplemental Material [14], we obtain the equation for the angular oscillation. The only new parameter is the distance $L_{1}$ between the rotation center $C$ and the top of the cylinder $D$ and we find that $K^{\prime}$ is related to the hydrodynamic damping time given by Eq. (26) by

$$
\eta K^{\prime}=\frac{3 I_{C}}{\pi\left[\left(L+L_{1}\right)^{3}-L_{1}^{3}\right]} \frac{1}{\tau^{h}},
$$

where $I_{C}$ is the moment of inertia of the pendulum, relative to the center of rotation $C$. The main issue is the calculation of $I_{C}$. When $\rho_{p}$ is homogeneously distributed over the whole length of the pendulum (this is the case of a solid or hollow cylinder), we find

$$
\frac{3 I_{C}}{\left(L+L_{1}\right)^{3}-L_{1}^{3}}=\frac{M}{L}\left[1+\frac{3}{4} \frac{\left(R^{2}+R_{\mathrm{int}}^{2}\right) L}{\left(L+L_{1}\right)^{3}-L_{1}^{3}}\right],
$$

which means that (27) and (28) differ only by a term less than $3 R^{2} /\left(2 L^{2}\right)$, hence less than $2 \times 10^{-3}$ for the $R \approx 19 \mathrm{~mm}$ pendulum and less than $1.5 \times 10^{-4}$ for the $R \approx 5 \mathrm{~mm}$ pendulum. 
TABLE II. For both pendulums, this table collects the values of the main parameters (mass $M$, cylinder length $L$, distance $L_{1}=C D$, and moment of inertia $I_{C}$ ) and of the quantity relating $\eta K^{\prime}$ to $1 / \tau^{h}$, following either Eq. (27) or Eq. (28).

\begin{tabular}{lcc}
\hline \hline Pendulum & 1 & 2 \\
\hline$M(\mathrm{~g})$ & 516.25 & 114.85 \\
$L(\mathrm{~mm})$ & 514.0 & 522.0 \\
$L_{1}(\mathrm{~mm})$ & 60.4 & 58.9 \\
$\left.10^{3} I_{C}(\mathrm{~kg} \mathrm{~m})^{2}\right)$ & 66.95 & 14.37 \\
$M / \pi L(\mathrm{~kg} / \mathrm{m})$ & 0.3197 & 0.0700 \\
$\frac{3 I_{C}}{\pi\left[\left(L+L_{1}\right)^{3}-L_{1}^{3}\right]}(\mathrm{kg} / \mathrm{m})$ & 0.3377 & 0.0701 \\
\hline \hline
\end{tabular}

A last difficulty subsists, however: the $R \approx 19 \mathrm{~mm}$ pendulum is not a simple hollow cylinder because two small plugs, of external radius equal to the cylinder internal radius $R^{\text {int }} \approx 16 \mathrm{~mm}$, are corking the two extremities. Those cylindrical plugs are necessary for attaching the spring at the top and the small magnet at the bottom. While it is easy to include their mass in $M$, it is harder to calculate their influence on $I_{C}$. This we do in the Supplemental Material [14], and Table II gives the values of the quantity relating $\eta K^{\prime}$ to $1 / \tau^{h}$ : the value of this quantity deduced from Eq. (28) is $5.6 \%$ larger than the value given by Eq. (27). We have made a similar calculation for the $R \approx 5 \mathrm{~mm}$ pendulum which deviates slightly from a perfect cylinder and the effect is very small: the ratio of $\eta K^{\prime}$ to $1 / \tau^{h}$ increases by less than $0.2 \%$ when the deviations from a perfect cylinder are taken into account (see Table II).

\section{THE EXPERIMENTAL RESULTS AND THEIR ANALYSIS}

\section{A. Data treatment}

The shadow detector signal $V(t) \propto x(t)$ is filtered by a sliding fast Fourier transform over 2048 data points corresponding to $\approx 51 \mathrm{~s}$ and we get the amplitude $a(t)$ as a function of the time $t$ (Fig. 3). It is easy to prove [18] that, in the case of an exponential decay, this filter does not modify the damping time constant. However, the set-up residual vibrations excite the pendulum [23] so that, at the end of the decay, $a(t)$ does not tend toward 0, as predicted by Eq. (24), but fluctuates with values $\approx 1 \mu \mathrm{m}$. The decay is exponential with a good accuracy from the initial amplitude $a(0) \approx 0.5 \mathrm{~mm}$ down to a $a(t) \approx 5 \mu \mathrm{m}$. We fit the logarithm of $a(t)$, using Eq. (24): this choice enhances the weight of the data at large $t$ but we must limit the $t$ range so that the vibration-induced fluctuations of $a(t)$ do not increase the damping time. For each record, the statistical uncertainty on the fitted $\tau$ value is of the order of $1 \mathrm{~s}$ but the dispersion over a set of similar records is larger: we use the average of these individual measurements as the experimental value noted $\tau^{\text {expt }}$ and, from the dispersion of these individual measurements, we estimate that its uncertainty is equal to $2 \%$. These values and their uncertainties are collected in the Supplemental Material [14]: they cover the 800-3500 s range for the $R \approx 5 \mathrm{~mm}$ pendulum and the $1100-5100 \mathrm{~s}$ range for the $R \approx 19 \mathrm{~mm}$ pendulum.

\section{B. Uncertainty of the measurements of $K^{\prime}$}

According to (28), to obtain the measured value $K^{\text {expt }}$ from $\tau^{\text {expt }}$ we must know the air viscosity $\eta$ and to present $K^{\text {expt }}$ in the form $K^{\text {expt }}=a+b(R / \delta)$ [as suggested by (3), (5), and (17)] we must know the air mass per unit volume $\rho$ because of its role in the viscous penetration depth. Both $\rho$ and $\eta$ are functions of the pressure, temperature, and the water molar fraction $x_{\mathrm{H}_{2} \mathrm{O}}$. While we control the pressure inside the pressurized tank, we have almost no control on the room temperature near $295 \mathrm{~K}$ and no control on the humidity of the ambient air. We just can say that a $\pm 2 \mathrm{~K}$ temperature variation (the maximum we observed during a series of experiments) leads to a $\mp 0.68 \%$ variation of 


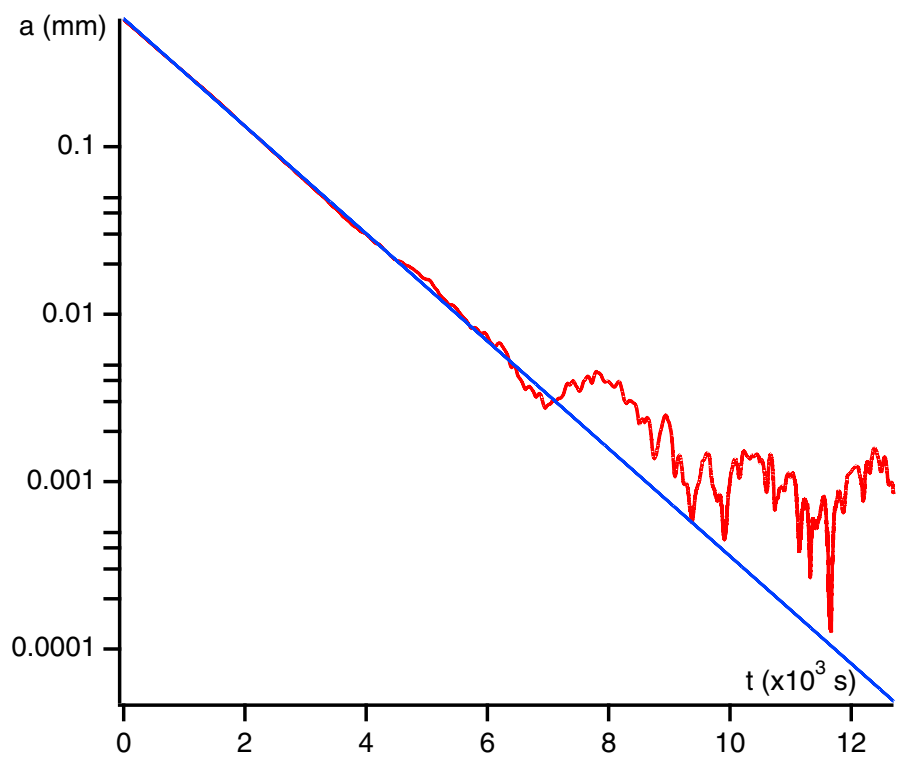

FIG. 3. Semilogarithmic plot of the amplitude $a(t)$ in millimeters as a function of $t$ in seconds: measurements (solid red line) and their best fit (solid blue line). As shown by the good agreement of the fit with the measured data, the amplitude is well an exponential function of time up to $t \approx 6.5 \times 10^{3} \mathrm{~s}$, i.e., for about $5 \tau^{\text {expt }}$ where the measured value of the damping time constant is $\tau^{\text {expt }} \approx 1334 \mathrm{~s}$. Cylinder radius $R \approx 19 \mathrm{~mm}$; pressure $P \approx 665$ mbar.

$\rho$ and a $\pm 0.53 \%$ variation of $\eta$ [24]. And when $x_{\mathrm{H}_{2} \mathrm{O}}$ increases from 0 to 0.033 (the saturation limit at $298 \mathrm{~K}$ and $P=1000$ mbar), $\eta$ decreases by $0.4 \%$ [25] while $\rho$ decreases by $1.3 \%$. Given those values, we estimate to about $1 \%$ the additional uncertainty on $K^{\prime}$ and we add this uncertainty to the $2 \%$ statistical uncertainty on $\tau^{\text {expt }}$. The relative uncertainty on $\delta$ is estimated to about $1 \%$ which is smaller than the symbols used in Fig. 4.

\section{COMPARISON OF OUR EXPERIMENTAL RESULTS WITH THEORY}

Figure 4 presents a plot of the measured $K^{\prime}$ as a function of $R / \delta$ for each of the two pendulums. A large fraction of the domain of $R / \delta$ we have explored belongs to the high-frequency limit for which prediction (17) holds. We used Loewenberg's numerical data [3,4] for our two pendulums and complete them with the Stokes result to give the expected values

$$
\begin{aligned}
& K^{\prime}=2.008 \frac{R}{\delta}+1.609, \quad \phi=13.3, \\
& K^{\prime}=2.001 \frac{R}{\delta}+1.433, \quad \phi=52.0, \\
& K^{\prime}=2 \frac{R}{\delta}+1, \quad \phi=\infty \quad \text { (Stokes) }
\end{aligned}
$$

Considering Fig. 4 , it is clear that the $\phi=13.3(R \approx 19 \mathrm{~mm})$ pendulum gives results in agreement with (29) but the $\phi=52.0(R \approx 5 \mathrm{~mm})$ pendulum gives results which are much closer to Stokes' prediction than to $(30)$.

It is possible that the steady drag coefficient $r_{\perp}^{0}(\phi)$ decreases with the aspect ratio a little bit faster than the logarithmic decrease suggested by Loewenberg in (14). And we remark that the more precise Stokes prediction, as given in (5), predicts for $\phi=\infty$ a coefficient $K^{\prime}$ a little bit smaller than 

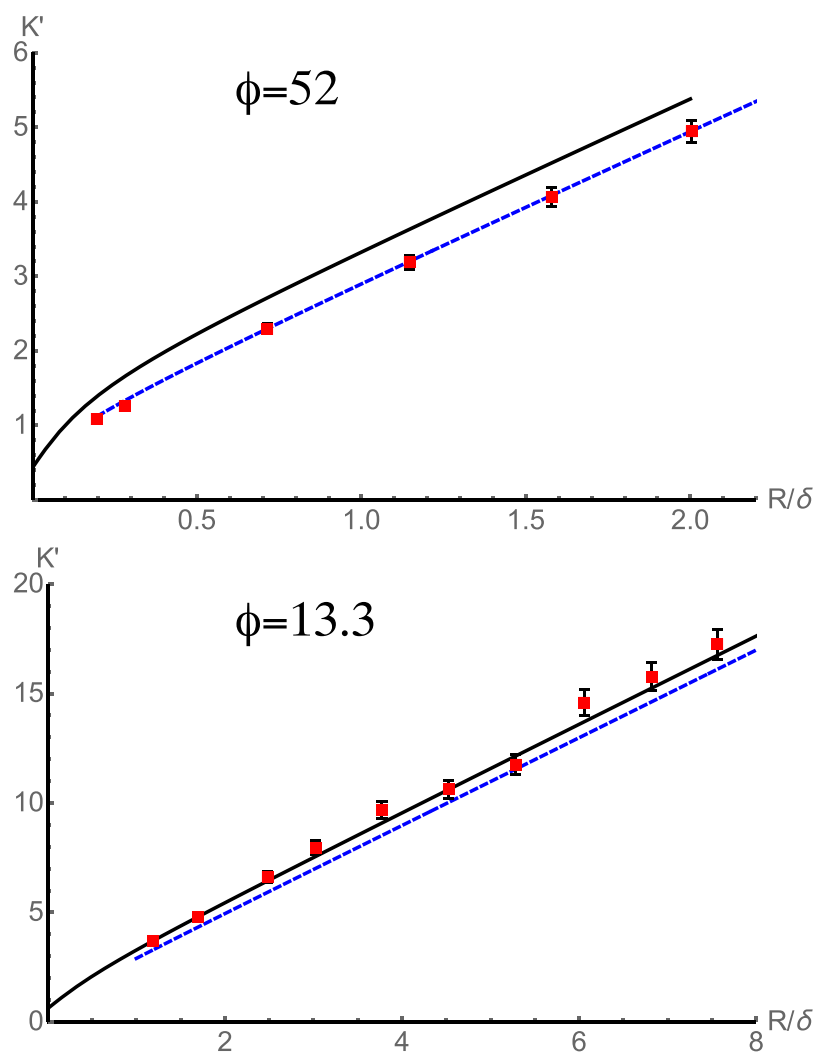

FIG. 4. Plot of $K^{\prime}$ as a function of the ratio $R / \delta$. The measured values $K^{\text {expt }}$ are represented by red squares. The dashed blue curve is Stokes' value given by Eq. (5) while the solid black curve represents the finite-cylinder value given by Eq. (12) (these three values are listed in Tables VII and VIII of the Supplemental Material [14]). Upper panel: data of the $R \approx 5 \mathrm{~mm}, \phi=52$ pendulum; lower panel: data of the $R \approx 19 \mathrm{~mm}, \phi=13.3$ pendulum.

$1+2 R / \delta$ in the high-frequency limit. But we must say we could not find a crystal-clear explanation for why the results for the $\phi=52$ pendulum are so close to what is expected for $\phi=\infty$.

\section{CAN EXPERIMENTS AT FINITE Re AGREE WITH THEORY AT Re $=0$ ?}

Many experimental tests have concluded that the force exerted by the fluid on cylinders in oscillating motion $[7,8,10,12,13]$ is in agreement with Stokes' prediction [with a relative uncertainty of order $\pm(10-20) \%$, however] and this agreement extends up to $\operatorname{Re} \approx 400$. Since Stokes' force on an infinite oscillating cylinder was obtained for $\operatorname{Re}=0$, the experimental agreement is rather puzzling and needs some comments. A first remark concerns the aspect ratio: it is clear that when $\phi>10$, as was the case of all previous experiments, the predictions for finite-length cylinders are close to those for an infinite cylinder [cf. Eq. (17) and the numerical values (29) and (30)] above all when $R / \delta>2$. The second remark concerns the Reynolds number: the nondimensional (incompressible) Navier-Stokes equation writes

$$
\operatorname{St} \frac{\partial \mathbf{u}}{\partial t}+\operatorname{Re}(\mathbf{u} \cdot \nabla) \mathbf{u}=-\nabla p+\nabla^{2} \mathbf{u} .
$$




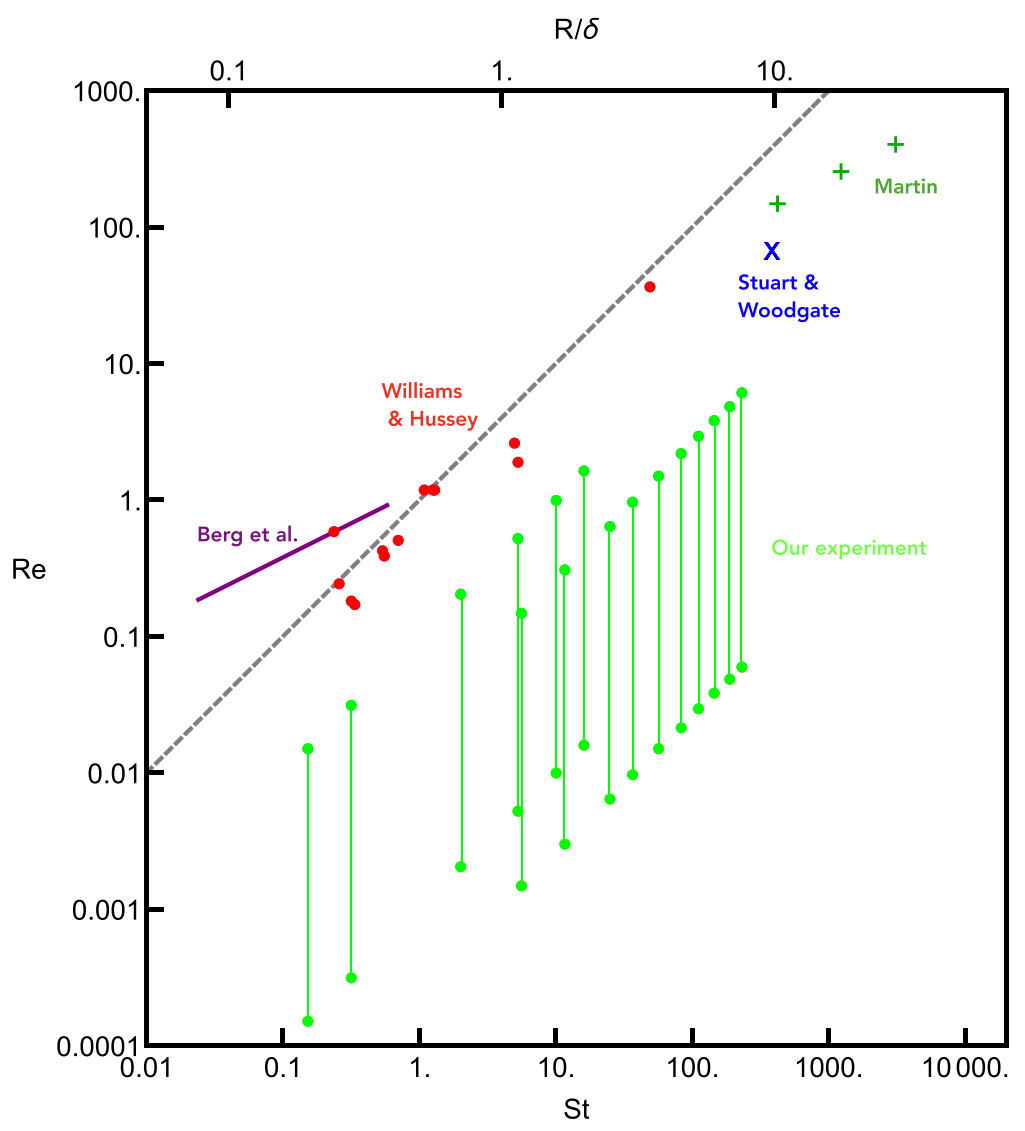

FIG. 5. Position of our experiments as well as previous ones in the Re-St plane (the upper scale gives $R / \delta$ ): (i) the violet solid line represents the results of Berg et al. [13] as discussed in the Supplemental Material [14]; (ii) the red bullets represent the results of Williams and Hussey [8]; and (iii) the green + signs represent the results of Martin [12] and the blue X represents the result of Stuart and Woodgate [10]. The green bullets, connected by green lines, represent the initial and final values of the Reynolds number during our experiments. Finally, the dashed gray line $\mathrm{Re}=\mathrm{St}$ suggests that most of the previous experiments agreeing with the Stokes predictions are far from satisfying condition (31).

One can expect the nonlinear force to have a negligible role as compared to the unsteady force when

$$
\mathrm{Re} \ll \text { St. }
$$

In other words, when an experiment satisfies the above condition, it can possibly check theoretical predictions obtained for $\operatorname{Re}=0$. For an oscillating cylinder the most widely accepted nondimensional numbers are

$$
\mathrm{St} \propto \frac{\rho \omega u}{\mu u / R^{2}} \propto \frac{R^{2}}{\delta^{2}}, \quad \operatorname{Re} \propto \frac{\rho u^{2} / R}{\mu u / R^{2}} \propto \frac{R^{2}}{\delta^{2}} \frac{x_{0}}{R}
$$

so that condition (31) on the Reynolds number is transformed into a condition on the oscillation amplitude

$$
x_{0} \ll R \text {. }
$$

Some investigators [13] used the above definition of the Stokes number, yet with a different definition of the Reynolds number. They assumed that the characteristic length of the inertial force 
was $\delta$ and not $R$. As a consequence $\operatorname{Re} \approx \operatorname{St}\left(x_{0} / \delta\right)$ and condition (31) implies

$$
x_{0} \ll \delta \text {. }
$$

Whatever is the correct condition on the oscillation amplitude, it remains that (31) must be fulfilled. For that reason we localize ours as well as previous experiments in a $(\mathrm{Re}, \mathrm{St})$ frame represented in Fig. 5 while the related $x_{0} / R$ or $x_{0} / \delta$ values can be found in Table I. As a mere guide for the eye, we also plotted in Fig. 5 the line $\mathrm{Re}=\mathrm{St}$. That all previous experiments could claim an agreement with the Stokesian prediction suggests that $\mathrm{Re}<\mathrm{St}$ can be used instead of the more stringent condition (31). But it is also clear from Fig. 5 that our experiments are definitely those which were operated with the smallest $\mathrm{Re} / \mathrm{St}$ ratio, namely, $\mathrm{Re} / \mathrm{St}<0.1$.

\section{CONCLUSION}

While many of the experiments on the unsteady forces in the Stokes regime were performed with micrometer particles in a liquid [26,27], the present experiments suggest it is also possible to check those forces with a macroscopic object, like a pendulum moving in a gas. Our experiments cover wide ranges of the Reynolds and Stokes numbers, with $0.2<\mathrm{St}<230.0$ and $\mathrm{Re} / \mathrm{St}<0.1$. The main difficulty concerns the amplitude of the oscillations which must be very small as compared to the pendulum radius. Thanks to the submicrometer sensitivity of the shadow detector used to record the pendulum oscillation, our measurements prove that the damping is exponential and from the damping relaxation time we have deduced the unsteady drag coefficient $K^{\prime}$. Our measurements with the $R \approx 19 \mathrm{~mm}$ pendulum (aspect ratio $\phi=13.3$ ) are in agreement with the finite-length cylinder theory for $K^{\prime}$ while our measurements with the $R \approx 5 \mathrm{~mm}$ pendulum (aspect ratio $\phi=52.0$ ) are much closer to Stokes' theory $(\phi=\infty)$ than to the finite-length cylinder theory. We could not find any explanation for that unexpected behavior.

\section{ACKNOWLEDGMENTS}

We want to thank D. Castex, E. Panader, S. Faure, L. Polizzi, and W. Volondat for their contributions to the experiment. We have benefited from very helpful discussions with P. Ern, M. Nicolas, and R. F. Berg. We also thank our anonymous referees for their numerous questions and suggestions which have helped us improve our paper. Financial support from CNRS INP, CNRS MI DEFI, and Université P. Sabatier is gratefully acknowledged.

[1] G. G. Stokes, On the Effect of the Internal Friction of Fluids on the Motion of Pendulums, Transactions of the Cambridge Philosophical Society Vol. IX, Part II, 8-106 (Cambridge University Press, Cambridge, UK, 1851).

[2] C. J. Lawrence and S. Weinbaum, The unsteady force on a body at low Reynolds number; the axisymmetric motion of a spheroid, J. Fluid Mech. 189, 463 (1988).

[3] M. Loewenberg, Stokes resistance, added mass, and Basset force for arbitrarily oriented, finite-length cylinders, Phys. Fluids A 5, 765 (1993).

[4] M. Loewenberg, The unsteady Stokes resistance of arbitrarily oriented, finite-length cylinders, Phys. Fluids A 5, 3004 (1993)

[5] C. A. Coulomb, Expériences destinées à déterminer la cohérence des fluides et les lois de leur résistance dans les mouvements très lents, Mém. l'Inst. Natl. Sci. Arts 3, 246 (1800).

[6] H. Tomlinson, The coefficient of viscosity of air, Philos. Trans. 177, 767 (1886).

[7] R. E. Williams, Oscillating cylinders and the Stokes paradox, Ph.D., Louisiana State University, LSU Historical Dissertations and Theses, 1972.

[8] R. E. Williams and R. G. Hussey, Oscillating cylinders and the Stokes paradox, Phys. Fluids 15, 2083 (1972); Erratum: Oscillating cylinders and the Stokes' paradox, 19, 1652 (1976). 
[9] F. Baily, On the correction of a pendulum for the reduction to a vacuum: Together with remarks on some anomalies observed in pendulum experiments, Philos. Trans. R. Soc. London 122, 399 (1832).

[10] J. T. Stuart and L. Woodgate, Experimental determination of the aerodynamic damping on a vibrating circular cylinder, Philos. Mag. 46, 40 (1955).

[11] L. R. Laird, On the period of a wire vibrating in a liquid, Phys. Rev (Ser. I) 7, 102 (1898).

[12] H. Martin, Uber tonhöhe und dämpfung der schwingungen von saiten in verschiedenen flüssigkeiten, Ann. Phys. (Leipzig, Ger.) 4, 627 (1925).

[13] R. F. Berg, M. Yao, and C. H. Panzarella, Hydrodynamic force on a cylinder oscillating at low frequency, Report No. NASA/CR 2007-215050.

[14] See Supplemental Material at http://link.aps.org/supplemental/10.1103/PhysRevFluids.6.104101 for the values of Loewenberg parameters for finite cylinders with a large aspect ratio, for the description of the pendulums and the calculation of their dynamical parameters, for the connection between the damping time constant and the friction force constant, for the measured values of these quantities, and for a discussion of previous experiments.

[15] J. T. Stuart, in Laminar Boundary Layers, edited by L. Rosenhead (Oxford University Press, New York, 1963), Chap. VII, pp. 347-408.

[16] R. G. Hussey and P. Vujacic, Damping correction for oscillating cylinder and sphere, Phys. Fluids 10, 96 (1967).

[17] G. Dolfo, D. Castex, and J. Vigué, Damping mechanisms of a pendulum, Eur. J. Phys. 37, 065004 (2016).

[18] G. Dolfo, J. Vigué, and D. Lhuillier, Experimental test of unsteady Stokes' drag force on a sphere, Exp. Fluids 61, 97 (2020).

[19] G. Dolfo and J. Vigué, A more accurate theory of a flexible-beam pendulum, Am. J. Phys. 83, 525 (2015).

[20] C. Zener, Internal Friction in Solids. I. Theory of Internal Friction in Reeds, Phys. Rev. 52, 230 (1937).

[21] C. Zener, Internal Friction in Solids. II. General Theory of Thermoelastic Internal Friction, Phys. Rev. 53, 90 (1938).

[22] G. Cagnoli, L. Gammaitoni, J. Hough, J. Kovalik, S. McIntosh, M. Punturo, and S. Rowan, Very High $Q$ Measurements on a Fused Silica Monolithic Pendulum for Use in Enhanced Gravity Wave Detectors, Phys. Rev. Lett. 85, 2442 (2000).

[23] D. Paget, J. Winterflood, Li Ju, and D. Blair, Improved technique for measuring high pendulum $Q$-factors, Meas. Sci. Technol. 13, 218 (2002).

[24] E. W. Lemmon and R. T. Jacobsen, Viscosity and thermal conductivity equations for nitrogen, oxygen, argon, and air, Int. J. Thermophys. 25, 21 (2004) and NIST software RefProp 9.0 (2010).

[25] J. Kestin and J. H. Whitelaw, The viscosity of dry and humid air, Int. J. Heat Mass Transfer 7, 1245 (1964).

[26] J. R. Allegra and S. A. Hawley, Attenuation of sound in suspensions and emulsions: Theory and experiments, J. Acoust. Soc. Am. 51, 1545 (1971).

[27] R. W. O’Brien, The electroacoustic equations for a colloidal suspension, J. Fluid Mech. 212, 81 (1990). 